\title{
Continuous high-repetition-rate operation of collisional soft-x-ray lasers with solid targets
}

\author{
A. Weith, M. A. Larotonda, Y. Wang, B. M. Luther, D. Alessi, M. C. Marconi, and J. J. Rocca \\ NSF Center for Extreme Ultraviolet Science and Technology, Colorado State University, \\ Fort Collins, Colorado 80523
}

\author{
J. Dunn \\ Lawrence Livermore National Laboratory, Livermore, California 94550
}

\begin{abstract}
Received April 18, 2006; revised April 18, 2006; accepted April 18, 2006; posted April 20, 2006 (Doc. ID 66358) We have generated a laser average output power of $2 \mu \mathrm{W}$ at a wavelength of $13.9 \mathrm{~nm}$ by operating a tabletop laser-pumped Ni-like $\mathrm{Ag}$ laser at a $5 \mathrm{~Hz}$ repetition rate, using a solid helicoidal target that is continuously rotated and advanced to renew the target surface between shots. More than $2 \times 10^{4}$ soft-x-ray laser shots were obtained by using a single target. Similar results were obtained at $13.2 \mathrm{~nm}$ in Ni-like Cd with a Cdcoated target. This scheme will allow uninterrupted operation of laser-pumped tabletop collisional soft-x-ray lasers at a repetition rate of $10 \mathrm{~Hz}$ for a period of hours, enabling the generation of continuous high average soft-x-ray powers for applications. (C) 2006 Optical Society of America

OCIS code: 140.7240.
\end{abstract}

Prolonged repetitive operation of pulsed lasers at optical wavelengths has been available for several decades and has made possible the implementation of numerous applications requiring intense pulses of coherent infrared, visible, and ultraviolet light. To extend and develop these applications at shorter wavelength requires high repetition-rate operation of soft$\mathrm{x}$-ray laser amplifiers in the gain-saturation regime. A renewable gain medium that can allow the uninterrupted generation of long series of laser shots is essential. Capillary discharge lasers based on the excitation of a gas by a fast discharge current pulse were the first soft-x-ray lasers to achieve prolonged operation in the gain-saturated regime at a repetition rate of up to $10 \mathrm{~Hz}^{1}{ }^{1}$ This made possible the use of a Nelike $\mathrm{Ar}$ laser emitting at $46.9 \mathrm{~nm}$ in several applications. ${ }^{2}$ Soft-x-ray lasers based on optical field ionization of gaseous targets emitting at wavelengths longer than $30 \mathrm{~nm}$ have also been demonstrated to operate repetitively in the gain-saturated regime. ${ }^{3,4}$ Several other experiments have demonstrated soft-xray laser amplification at multihertz repetition rates but without achieving the gain-saturated amplification levels necessary to produce significant average powers. ${ }^{5,6}$

Transient collisional electron excitation of plasmas by normal incidence irradiation of solid targets with a nanosecond pulse followed by a picosecond pump pulse of 3-10 J energy has produced several saturated lasers in the $12-33 \mathrm{~nm}$ range, at repetition rates of one shot every several minutes. ${ }^{7,8}$ Recently, the laser pump energy required for obtaining gainsaturated operation of soft-x-ray lasers was significantly reduced by directing the picosecond pump pulse at a grazing angle of incidence into the precreated plasma. ${ }^{9-14}$ The use of picosecond-duration pump laser pulses with energies up to $1 \mathrm{~J}$ impinging at grazing incidence angles from $14^{\circ}$ to $23^{\circ}$ resulted in the demonstration of gain-saturated lasers in transitions of Ni-like ions ${ }^{9-12}$ and Ne-like ions ${ }^{13}$ at wave- lengths as short as $13.2 \mathrm{~nm}$ for Ni-like Cd. These lasers were demonstrated to operate at repetition rates of $5-10 \mathrm{~Hz}$ by pumping polished slab targets of the selected laser element. The number of laser shots that can be obtained by pumping the same target surface depends on the material. While for Ni-like Mo up to 20-30 laser shots can be obtained without moving the target between shots, for Ni-like Ag or Nilike Cd the laser output intensity is observed to degrade after only $2-3$ shots on the same target surface. This quick degradation raises the question whether these materials are suitable for extended continuous operation.

Herein we report the quasi-continuous operation of a saturated $13.9 \mathrm{~nm}$ Ni-like Ag soft-x-ray laser at a $5 \mathrm{~Hz}$ repetition rate, using a scheme that will allow uninterrupted soft-x-ray laser output at a repetition rate of $10 \mathrm{~Hz}$ for a period of hours. An average power of $2 \mu \mathrm{W}$ and a high average spectral brightness of $1.3 \times 10^{13}$ photons $\mathrm{mm}^{-2} \mathrm{mrad}^{-2} \mathrm{~s}^{-1} \quad(0.1 \%$ bandwidth) $)^{-1}$ at $13.9 \mathrm{~nm}$ was demonstrated. The laser makes use of a Ag-coated solid helicoidal target that is continuously rotated and advanced to renew the target surface between shots, enabling the prolonged generation of high average power for applications. More than $2 \times 10^{4}$ laser shots at a single target with a useful spiral perimeter length of $96 \mathrm{~cm}$ rotated at a tangential speed of $0.2 \mathrm{~mm} \mathrm{~s}^{-1}$ were achieved at a $5 \mathrm{~Hz}$ repetition rate. An increase in the number of laser shots supported by the target to $\sim 5 \times 10^{4}$ was achieved by reducing the tangential rotation velocity to $0.1 \mathrm{~mm} \mathrm{~s}^{-1}$ at the expense of reducing the soft-xray laser average power by $\sim 20 \%$. This setup will allow uninterrupted saturated operation of laserpumped tabletop collisional soft-x-ray lasers at a repetition rate of $10 \mathrm{~Hz}$ for a period of $\sim 2 \mathrm{~h}$.

The plasma that constitutes the soft-x-ray laser gain medium was generated by focusing the $800 \mathrm{~nm}$ wavelength beam of a Ti:sapphire pump laser onto the outermost surface of the helicoidal target to form 

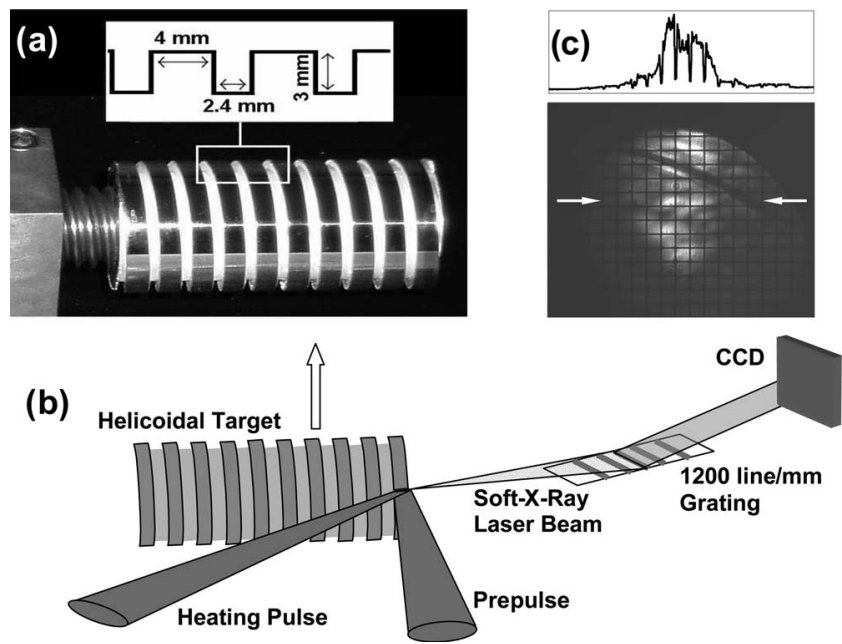

Fig. 1. (a) Schematic diagram of the soft-x-ray laser experimental setup with a helicoidal soft-x-ray laser target for high-repetition-rate soft-x ray laser operation. (b) Photograph of the helicoidal target. For optimized operation at a $5 \mathrm{~Hz}$ repetition rate in the $13.9 \mathrm{~nm}$ line of Ni-like $\mathrm{Ag}$ the target was rotated at a tangential velocity of $0.2 \mathrm{~mm} \mathrm{~s}^{-1}$. (c) Far-field image of the laser output, with an intensity profile obtained at the indicated position. The square structure is produced by the supporting mesh of the $\mathrm{Zr}$ filter.

a line focus $30 \mu \mathrm{m}$ wide and $4 \mathrm{~mm}$ long. A schematic diagram of the experimental setup and a photograph of the helicoidal target are shown in Fig. 1. The target consists of a solid cylindrical body of $30 \mathrm{~mm}$ diameter and $63 \mathrm{~mm}$ length whose outer surface was machined to constitute a helicoid of rectangular cross section with a $4 \mathrm{~mm}$ wide top surface and a $6.4 \mathrm{~mm}$ period. The target was made of electrolytic copper and was electroplated with a $\sim 20 \mu \mathrm{m}$ thick Ag coating. It was mounted on a motorized screw that had a pitch designed to continuously rotate and advance the target while making the horizontal position of the $4 \mathrm{~mm}$ wide stripe look stationary to the pump laser beam. A stepper motor was used to rotate the screw and advance it with respect to a stationary nut, renewing the target surface irradiated by the pump beam.

The gain medium plasma was formed by irradiating the outer surface of the Ag-coated helicoidal target at near-normal incidence with a sequence of an early prepulse of $120 \mathrm{ps}$ duration and $\sim 10 \mathrm{~mJ}$ energy, followed after $\sim 5 \mathrm{~ns}$ by a main prepulse of the same duration and $\sim 350 \mathrm{~mJ}$ energy, which in turn was followed after a selected delay of 300 ps (100 ps for Cd) by an $8 \mathrm{ps}$ duration heating pulse of $\sim 1 \mathrm{~J}$ energy impinging at an optimized grazing incidence angle of $23^{\circ}$. The prepulse beam was focused into a $4.1 \mathrm{~mm}$ long $\times 30 \mu \mathrm{m}$ wide line by a combination of an $f=67.5 \mathrm{~cm}$ spherical lens and an $f=200 \mathrm{~cm}$ cylindrical lens. The ps heating pulse was also focused into a $30 \mu \mathrm{m}$ FWHM wide, $4.1 \mathrm{~mm}$ long line on the target by use of an $f=76.2 \mathrm{~cm}$ focal length multilayercoated parabolic mirror placed at $7^{\circ}$ from normal incidence. The plasma emission was monitored using a flat field spectrograph composed of a 1200 line $/ \mathrm{mm}$ Au-coated spherical grating with a variably spaced ruling positioned at $87^{\circ}$ and of a $2.76 \mathrm{~cm} \times 2.76 \mathrm{~cm}$, back-illuminated CCD detector array placed in the image plane of the grating located $48 \mathrm{~cm}$ from the target. The plasma emission was filtered with a $0.34 \mu \mathrm{m}$ thick $\mathrm{Zr}$ film and was attenuated with a set of metallic meshes with a total transmission of $3.6 \%$ to avoid saturation of the CCD detector.

Figure 2 shows a single-shot spectrum of the axial emission of a $\mathrm{Ag}$ plasma generated by heating the helicoidal target. The $13.9 \mathrm{~nm}$ line of Ni-like Ag is observed to completely dominate the spectrum. The most intense laser pulses were measured to have an energy of $850 \mathrm{~nJ}$. The laser intensity is very similar to that obtained using a flat, polished Ag slab target, for which the measured gain-length product of 16.8 and the observed output intensity indicate operation in the gain-saturated regime. ${ }^{11}$ The beam quality is illustrated by the far-field pattern shown in Fig. 1(c). Shooting a second shot onto the same Ag target surface resulted in a decreased laser output intensity. Full recovery of the laser intensity was observed when the target was rotated to displace the ablated target surface by at least $40 \mu \mathrm{m}$. This induced us to select a tangential target velocity of $0.2 \mathrm{~mm} \mathrm{~s}^{-1}$ for the soft-x-ray laser operation at a $5 \mathrm{~Hz}$ repetition rate. An increase of the rotation velocity beyond this point did not cause any significant increase in laser output energy. In contrast, a reduction of the tangential velocity to $0.1 \mathrm{~mm} \mathrm{~s}^{-1}$, which doubles the numbers of shots that can be obtained from a single target at the expense of partially overlapping the target regions directly irradiated by the laser beam in two contiguous shots, was observed to reduce the soft-xray laser output energy per pulse by $\sim 20 \%$.

Figure 3 shows three contiguous series of 250 laser shots, each obtained at intervals of $5 \mathrm{~min}$. The average laser pulse energy for the three series is $400 \mathrm{~nJ}$, corresponding to an average power of $2 \mu \mathrm{W}$, and the shot-to-shot variation is characterized by a standard deviation of $19 \%$. Longer series of shots showed a continuous decrease of the output pulse energy after the first $\sim 250$ shots. The decrease in laser pulse energy was traced to degradation of the pump laser beam wavefront and the line focus quality owing to thermal loading of the replica diffraction gratings of the pulse compressor. As illustrated in Fig. 3, an interval of 4-5 min between consecutive series of 250 shots allows for full recovery of the laser intensity. The use of master diffraction gratings should readily

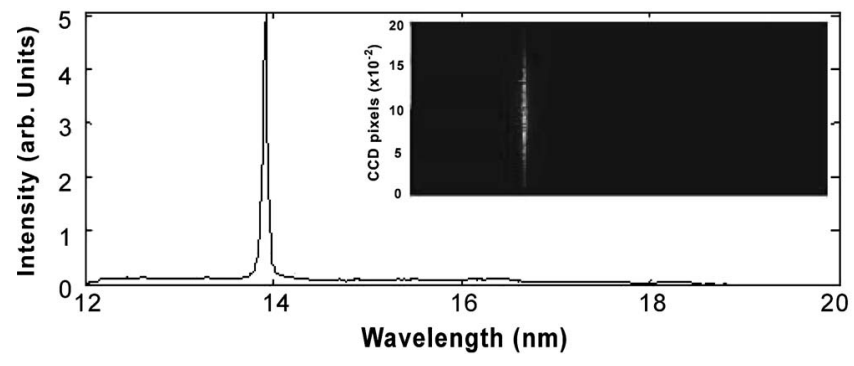

Fig. 2. Spectrum of the emission from a Ni-like Ag laser operating at a $5 \mathrm{~Hz}$ repetition rate with the rotating helicoidal target. The $13.9 \mathrm{~nm}$ line completely dominates the spectrum. Inset, image of the spectrum captured on the CCD. 


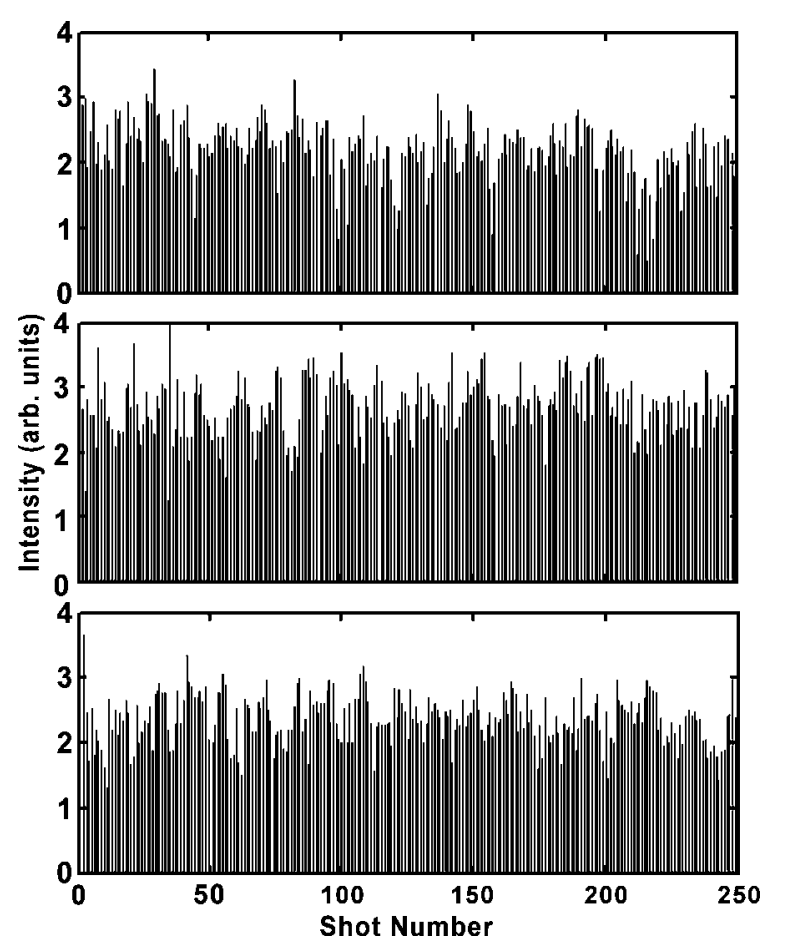

Fig. 3. Three contiguous series of 250 laser shots at $5 \mathrm{~Hz}$ repetition for a $\lambda=13.9 \mathrm{~nm}$ Ni-like $\mathrm{Ag}$ laser obtained at 5 min intervals. The average power is $\sim 2 \mu \mathrm{W}$.

allow uninterrupted operation of the $5 \mathrm{~Hz}$ laser for more than $5 \times 10^{4}$ laser shots, corresponding to more than $2 \mathrm{~h}$ of continuous laser operation. The straightforward increase of the target length will allow several hours of uninterrupted $10 \mathrm{~Hz}$ repetition rate laser operation and potentially double the average power to $4 \mu \mathrm{W}$ by use of $0.2 \mathrm{~mm} \mathrm{~s}^{-1}$ speeds. Laser operation at other soft-x-ray wavelengths can be readily obtained by selecting a coating that allows lasing in transitions of other Ni-like or Ne-like ions. Similar results were obtained for the $13.2 \mathrm{~nm}$ line of Ni-like $\mathrm{Cd}$ by coating the target with $\mathrm{Cd}$. A laser average output power of $\sim 1 \mu \mathrm{W}$ was generated in this line as the result of operation at $5 \mathrm{~Hz}$ repetition with the target moving at $0.2 \mathrm{~mm} \mathrm{~s}^{-1}$.

This x-ray laser source has a combination of both high average power and high average spectral brightness as a result of the $5-10 \mathrm{~Hz}$ repetition rate described here. The $2 \mu \mathrm{W}$ average power operation is equivalent to $1.5 \times 10^{11}$ photons/s, while the energy of the most intense pulses approaches $1 \mu \mathrm{J}$. The estimated average brightness of the source, 1.3 $\times 10^{13}$ photons $\mathrm{mm}^{-2} \mathrm{mrad}^{-2} \mathrm{~s}^{-1}(0.1 \% \text { bandwidth })^{-1}$, is similar to that of some synchrotron bending magnets operating at the same photon wavelength. Simultaneously, as a result of the measured short pulse duration of $5 \mathrm{ps}$ (FWHM) for the $\mathrm{Ag} 13.9 \mathrm{~nm}$ line, a high peak power of $0.1-0.2 \mathrm{MW}$ and a peak spectral brightness of approximately 0.5-1 $\times 10^{24}$ photons $\mathrm{mm}^{-2} \mathrm{mrad}^{-2} \mathrm{~s}^{-1}(0.1 \% \text { bandwidth })^{-1}$ can be achieved. This peak spectral brightness exceeds by 2 to 3 orders of magnitude that of undulators operating at this wavelength.
In summary, we have demonstrated a scheme that allows, for the first time to our knowledge, the operation of high-repetition-rate laser-pumped transient collisional lasers emitting near a range of $100 \mathrm{eV}$ photons for periods of hours. The uninterrupted generation of high-average-power soft-x-ray laser radiation will enable numerous applications to be made.

This work was supported by the National Science Foundation (NSF) Center for Extreme Ultraviolet Science and Technology under NSF award EEC0310717. We gratefully acknowledge the support of the W. M. Keck Foundation. The work of J. Dunn was performed under the auspices of the U.S. Department of Energy by the University of California Lawrence Livermore National Laboratory under contract W-7405-Eng-48. J. Rocca's e-mail address is rocca@engr.colostate.edu.

\section{References}

1. B. R. Benware, C. D. Macchietto, C. H. Moreno, and J. J. Rocca, Phys. Rev. Lett. 81, 5804 (1998).

2. Several of these applications are discussed in J. J. Rocca, M. Frati, B. Benware, M. Seminario, J. Filevich, M. Marconi, K. Kanizay, A. Ozols, I. A. Artiukov, A. Vinogradov, and Y. A. Uspenskii, C. R. Acad. Sci. 1, 1065 (2000), and in references therein.

3. S. Sebban, R. Haroutunian, P. Balcou, G. Grillon, A. Rousse, S. Kazamias, T. Marin, J. P. Rousseau, L. Notebaert, M. Pittman, J. P. Chambaret, A. Antonetti, D. Hulin, D. Ross, A. Klisnick, A. Carillon, P. Jaegle, G. Jamelot, and J. F. Wyart, Phys. Rev. Lett. 86, 3004 (2001).

4. S. Sebban, T. Mocek, D. Ross, L. Upcraft, P. Balcou, R. Haroutunian, G. Grillon, B. Rus, A. Klisnick, A. Carillon, G. Jamelot, C. Valentin, A. Rousse, J. P. Rousseau, L. Notebaert, M. Pittman, and D. Hulin, Phys. Rev. Lett. 89, 253901 (2002).

5. D. V. Korobkin, C. H. Nam, S. Suckewer, and A. Golstov, Phys. Rev. Lett. 77, 5206 (1996).

6. T. Ozaki, R. A. Ganeev, A. Ishizawa, T. Kanai, and H. Kuroda, Phys. Rev. Lett. 89, 253902 (2002).

7. P. V. Nickles, V. N. Shlyaptsev, M. Kalachinkov, and M. Schnurer, Phys. Rev. Lett. 78, 2748 (1997).

8. J. Dunn, Y. Li, A. L. Osterheld, J. Nilsen, J. R. Hunter, and V. N. Shlyaptsev, Phys. Rev. Lett. 84, 4834 (2000).

9. R. Keenan, J. Dunn, P. K. Patel, D. F. Price, R. F. Smith, and V. N. Shlyaptsev, Phys. Rev. Lett. 94, 103901 (2005).

10. B. M. Luther, Y. Wang, M. A. Larotonda, D. Alessi, M. Berrill, M. C. Marconi, J. J. Rocca, and V. Shlyaptsev, Opt. Lett. 30, 165 (2005).

11. Y. Wang, M. A. Larotonda, B. M. Luther, D. Alessi, M. Berrill, J. J. Rocca, and V. N. Shlyaptsev, Phys. Rev. A 72, 053807 (2005).

12. J. J. Rocca, Y. Wang, M. A. Larotonda, B. M. Luther, M. Berrill, and D. Alessi, Opt. Lett. 30, 2581 (2005).

13. D. Alessi, B. M. Luther, Y. Wang, M. A. Larotonda, M. Berrill, and J. J. Rocca, Opt. Express 13, 2093 (2005).

14. M. A. Larotonda, B. M. Luther, Y. Wang, Y. Liu, D. Alessi, M. Berrill, A. Dummer, F. Brizuela, C. S. Menoni, M. C. Marconi, V. N. Shlyaptsev, J. Dunn, and J. J. Rocca, IEEE J. Sel. Top. Quantum Electron. 10, 1363 (2004). 